September 7, 2018

OITS-673

LBNL-43082

UCB-PTH-99/12

\title{
Signals of Supersymmetric Lepton Flavor Violation at the LHC \&
}

\author{
Kaustubh Agashe ${ }^{a, \text {, }}$ and Michael Graesser ${ }^{b \text {, f }}$ ? \\ ${ }^{a}$ Institute of Theoretical Science \\ 5203 University of Oregon Eugene OR 97403-5203 \\ b Theoretical Physics Group \\ Lawrence Berkeley National Laboratory \\ University of California \\ Berkeley CA 94720
}

\begin{abstract}
In a generic supersymmetric extension of the Standard Model, there will be lepton flavor violation at a neutral gaugino vertex due to misalignment between the lepton Yukawa couplings and the slepton soft masses. Sleptons produced at the LHC through the cascade decays of squarks and gluinos can give a sizable number of events with 4 leptons. This channel could give a clean signature of supersymmetric lepton flavor violation under conditions which are identified.
\end{abstract}

\footnotetext{
${ }^{1}$ A preliminary version of this work was presented by KA at the Higgs and Supersymmetry: Search and Discovery Conference at the University of Florida, Gainesville, March 8-11, 1999.

${ }^{2}$ This work is supported by DOE Grant DE-FG03-96ER40969.

${ }^{3}$ email: agashe@oregon.uoregon.edu.

${ }^{4}$ email: mlgraesser@lbl.gov.

${ }^{5}$ Supported by the Natural Sciences and Engineering Research Council of Canada.
} 


\section{Introduction}

In the supersymmetric Standard Model (SM), the quadratically divergent corrections to the Higgs $(\text { mass) })^{2}$ cancel due to supersymmetry (SUSY). The remaining corrections are logarithmically divergent, proportional to the SUSY breaking masses of the sparticles (the superpartners of the SM particles) and result in a negative Higgs (mass) ${ }^{2}$ due to the large top quark Yukawa coupling. Thus, the superpartners of the SM particles must have masses $\lesssim 1 \mathrm{TeV}$ in order for SUSY to solve the gauge hierarchy problem and lead to natural electroweak symmetry breaking.

With the sparticle masses at the weak scale, these new particles (especially gluinos and squarks) will be produced in significant amounts at the LHC. After the initial discovery of the sparticles, the focus will be on precision measurements of their masses and mixings just as, for example, the next step after the discovery of the heavy quarks was the measurement of their detailed properties. In this paper, a relatively clean signal at the LHC for detecting the mixing angle between the scalar partners of the charged leptons (the sleptons) is presented.

A flavor-violating signal is obtained from the production of real sleptons, followed by their oscillation into a different flavored slepton, and subsequent decay to a lepton. Some formulae for these oscillations are given in section 1.1. At a ee linear collider, the production of slepton pairs can then give $e \mu$ events with missing energy. This was studied in [1, 2]. Dilepton flavor and $\mathrm{CP}$ violating signals at the LHC and NLC were studied in [3]. At a hadron collider (the LHC), sleptons can be pair-produced by the Drell-Yan process giving the same signal. This was studied in [4, 5, and is a promising signal for large flavor mixing angles and when the SUSY background is known to be small. Real sleptons can also be produced at the LHC in the decays of the next-to-lightest neutralino $\left(\chi_{2}^{0}\right)$, which are mainly produced in the cascade decays of gluinos and squarks. In section 3.1, flavor violating dilepton events from $\chi_{2}^{0}$ decays are briefly considered. The production of $\chi_{2}^{0}$ pairs can give rise to events with 4 leptons, with the dramatic flavor violating signal identified by a $(3 e+\mu)$ or $(3 \mu+e)$ lepton signature, hard jets, no $b$-jets, and of course missing energy. This is discussed in section 3.2. Conditions on the supersymmetric spectrum that are favorable for the suppression of the dominant supersymmetric background, occuring from heavier neutralino/chargino and stop decays, are identified. Ideas for determining the remaining dominant supersymmetric background occuring from $\tau$ decays are also presented. These are all conveniently summarized in the Conclusion.

In section 3.2.1, a brief estimate of the expected 4-lepton signal at a generic point in SUSY parameter space is given. Next, in section [3.2.2, a particular LHC Point [8, 9] is considered. It is found that at this Point, a $5 \sigma$ discovery ( $2 \sigma$ exclusion) is obtained for a right-handed ( $\mathrm{RH}$ ) first and second generation mixing angle $\sin \theta_{R}>0.13\left(\sin \theta_{R}>0.08\right)$ with an integrated luminosity of $100 \mathrm{fb}^{-1}$ at low luminosity. The discovery potential at high luminosity is still optimistic though less quantitative, due to uncertainties in $\tau$-jet detection efficiencies and larger $b$-jet mistagging rates. In any case, the values for the mass splitting (between $\tilde{e}$ and $\tilde{\mu}$ ) that are favorable for the 
discovery of a signal satisfy the $\mu \rightarrow e \gamma$ bound even for a maximal mixing angle. Thus the LHC has the opportunity of probing mixing angles that are beyond the reach of the current $\mu \rightarrow e \gamma$ limit.

\subsection{Lepton Flavor Violation due to Slepton Mass Mixing}

To begin consider the lepton-slepton-neutral gaugino vertex with the leptons and sleptons in the gauge basis:

$$
\tilde{l}_{a}^{* \text { gauge }} l_{a}^{\text {gauge }} \chi^{0}
$$

where $a=1,2,3$ is a flavor index. Next perform a unitary transformation, $V$, on both $l_{g}$ and $\tilde{l}_{g}$ to go to the mass eigenstate basis for the l's:

$$
\tilde{l}_{\alpha}^{*} l_{\alpha} \chi^{0}
$$

In this basis the coupling remains diagonal in flavor space (now denoted by $\alpha$ ). In general, however, the slepton and lepton mass matrices are not related so that the same unitary matrix, $V$, may not diagonalize them both. In this general case, the slepton (mass) ${ }^{2}$ matrix in the basis

$\tilde{l}_{\alpha}$ is not diagonal. For example, even if the slepton (mass) ${ }^{2}$ matrix in the gauge basis $\tilde{l}_{a}^{\text {gauge }}$ of Eqn.(11) is diagonal but not $\propto \mathbf{1}$, it will have off-diagonal elements in the basis $\tilde{l}_{\alpha}$ of Eqn.(2). So, a further unitary transformation, $W$, is needed to rotate to the slepton mass basis. In this basis the slepton-lepton-gaugino vertex is:

$$
\tilde{l}_{i}^{*} l_{\alpha} W_{i \alpha} \chi^{0}
$$

So, in the mass basis for leptons and sleptons $\left(l_{\alpha}\right.$ and $\left.\tilde{l}_{i}\right)$ a mixing matrix $W \neq \mathbf{1}$ in general appears at the neutral gaugino-lepton-slepton vertex. This means that there is a coupling between, for example, $\tilde{e}$ (in the mass basis), $\mu$ and $\chi^{0}$ - this will be referred to as SUSY lepton flavor violation. The focus of this paper is the detection of this SUSY lepton flavor violation at the LHC.

The theoretical expectations for $W$ are varied. In models with broken flavor symmetries, it is expected that $W \sim V_{K M}$. In such cases a Cabibbo-like mixing angle for the first two generations and a $\Delta m / m$ close to the $\mu \rightarrow e \gamma$ bound is expected [6]. In contrast, in models of gauge-mediated supersymmetry breaking the dominant contribution to the soft masses is universal and it naively appears that there is no interesting flavor physics. There is, however, a subdominant flavor non-universal supergravity contribution. This likely results in large mixing angles [3]. The magnitude of $\Delta m / m$ depends on the supersymmetry breaking scale and while clearly model-dependent, could easily be $\sim \Gamma / m$ or larger, which is needed to give an observable flavor-violating signal at the LHC (this is discussed later in this section).

For simplicity, the case of $1-2$ mixing with mixing angle $\theta$ is discussed. In this case there are strong limits on the mixing angle and the $\tilde{e}-\tilde{\mu}$ mass splitting from lepton flavor changing processes. For example, $\mu \rightarrow e \gamma$ gives an important constraint. For degenerate left-handed 
sleptons, and with the LSP $\left(\chi_{1}^{0}\right)$ approximately bino-like $\left(\tilde{B}^{0}\right)$, the constraint on $\sin 2 \theta_{R}$ and the mass splitting $\Delta m$ between the right-handed sleptons is approximately

$$
\sin 2 \theta_{R}(\Delta m) / m \lesssim 0.01 \times \sqrt{\frac{B R(\mu \rightarrow e \gamma)}{4.9 \times 10^{-11}}} .
$$

(A more proper formula is given in Section 3.2.2).

Suppose a real selectron is produced in the basis of Eqn.(2) (say in association with an electron). Since $\tilde{e}(\alpha=1)$ is not a mass eigenstate, there is a probability that as it propagates it will convert to a $\tilde{\mu}(\alpha=2)$ and hence decay into a $\mu[2,3]$ :

$$
P\left(\tilde{e}_{\alpha=1} \rightarrow \mu \chi_{1}^{0}\right)=2 \sin ^{2} \theta \cos ^{2} \theta x
$$

where $x=(\Delta m)^{2} /\left((\Delta m)^{2}+\Gamma^{2}\right)$ is the quantum interference factor and assuming $B R(\tilde{l} \rightarrow$ $\left.l \chi_{1}^{0}\right)=1$. Here $\Gamma$ is the decay width of the slepton. Note that for $\Delta m \gtrsim \Gamma$ the interference effect can be neglected so that $x \sim 1$. In this case the oscillation probability can be large. Typically, $\Gamma \sim \alpha_{e m} m \sim 0.01 m$ so that $x \sim 1$ if $(\Delta m) / m \gtrsim 0.01$. This is close to the upper bound from the $\mu \rightarrow e \gamma$ limit, so there could be a suppression due to either $x$ or $\sin \theta$ [2, 3]. It is possible, however, that for a specific SUSY spectrum the decay width could be much smaller than this naive estimate, allowing for a larger range of $\Delta m / m$ consistent with the rare decay limit (even for large mixing angles) and $x \sim 1$ so that the oscillation signal is not suppressed $\square$.

Similarly, a neutralino can decay into $e^{+} \mu^{-}$or $e^{-} \mu^{+}$through an intermediate slepton:

$$
\chi_{2}^{0} \rightarrow \tilde{l}^{+} l^{-}, \tilde{l}^{-} l^{+} \rightarrow l^{+} l^{-} \chi_{1}^{0} .
$$

Using Eqn.(5) the rate for a flavor violating decay is

$$
B R\left(\chi_{2}^{0} \rightarrow e^{+} \mu^{-} \chi_{1}^{0}\right)=2 \sin ^{2} \theta \cos ^{2} \theta x \times B R\left(\chi_{2}^{0} \rightarrow \tilde{e}^{-} e^{+}, \tilde{\mu}^{+} \mu^{-}\right) .
$$

Here to simplify notation $B R\left(\chi_{2}^{0} \rightarrow \tilde{e}^{-} e^{+}, \tilde{\mu}^{+} \mu^{-}\right) \equiv B R\left(\chi_{2}^{0} \rightarrow \tilde{e}^{-} e^{+}\right)+B R\left(\chi_{2}^{0} \rightarrow \tilde{\mu}^{+} \mu^{-}\right)$. This notation will be used throughout the paper. Also, the BR on the right-side of Eqn.(7) is in the absence of any mixing. In the case of interest here of small mass splittings, $\Delta m \ll m$, the neutralino decay rate into selectrons or smuons are equal in the absence of any mixing. Next, in the absence of mixing,

$$
B R\left(\chi_{2}^{0} \rightarrow e^{+} e^{-} \chi_{1}^{0}\right)=2 B R\left(\chi_{2}^{0} \rightarrow \tilde{e}^{+} e^{-}\right)
$$

The factor of two occurs since $\chi_{2}^{0}$ may decay to és of both charges. This result and Eqn.(77) relates the flavor-violating and flavor-conserving decays:

$$
B R\left(\chi_{2}^{0} \rightarrow e^{+} \mu^{-} \chi_{1}^{0}\right)=2 \sin ^{2} \theta \cos ^{2} \theta x \times B R\left(\chi_{2}^{0} \rightarrow l^{+} l^{-} \chi_{1}^{0}\right),
$$

\footnotetext{
${ }^{6}$ In fact, this occurs at the LHC Point discussed in Section 3.2.2.
} 
where the BR on the right-side of the above equation is in the absence of mixing. Here $l$ is either $e$ or $\mu$. This result applies for $\chi_{2}^{0}$ decays to real sleptons, i.e., for $m_{\chi_{2}^{0}}>m_{\tilde{l}}$. For $m_{\chi_{2}^{0}}<m_{\tilde{l}}$, there is an additional suppression of $(\Delta m) / m$ in the decay amplitude due to the supersymmetric analog of the Glashow-Iliopoulos-Maiani (GIM) cancellation as in the case of $\mu \rightarrow e \gamma$, resulting in negligible $e \mu$ signal. So an observable $e \mu$ signal requires the production of real sleptons $\square$.

\section{Slepton Production by Drell-Yan Process}

One way to produce sleptons at a hadron collider is through the Drell-Yan process:

$$
p p(\text { or } \bar{p}) \stackrel{\gamma, Z}{\rightarrow} \tilde{l}^{*} \tilde{l} \rightarrow l^{+} l^{-} \chi_{1}^{0} \chi_{1}^{0}
$$

Thus the production of sleptons is identified by events with no jets, 2 hard isolated leptons and $\not p_{T}$, assuming that $\chi_{1}^{0}$ is stable or decays outside the detector. These events will be referred to as "flavor conserving" dilepton events.

There is a SM background to the signal from $W^{+} W^{-}$and $\bar{t} t$ production. These backgrounds are known, in principle. In [7] a set of kinematic cuts on the leptons, as well as a jet-veto, are found which sufficiently reduce these backgrounds. These cuts reduce the signal as well - of course, the reduction is much more for the background.

There is also a SUSY background from $p p \rightarrow \chi^{+} \chi^{-} \rightarrow W^{+} W^{-} \chi_{1}^{0} \chi_{1}^{0}$. This background depends on the model-dependent $\chi^{+} \chi^{-}$production cross section. But, for supergravity motivated parameter choices with $m_{\tilde{q}} \approx m_{\tilde{g}}$, this background can be sufficiently reduced by using the same cuts used to remove the SM background [7]. For example, from the analysis of [7] (see Table III of [6]) with $10(\mathrm{fb})^{-1}$ and for a slepton mass $\sim 100 \mathrm{GeV}$ there are $\sim 20$ signal events with no background events remaining after the cuts.

Actually, a clever method [5] for detecting the sleptons is to form the asymmetry $A_{F}=$ $N\left(e^{+} e^{-}+\mu^{+} \mu^{-}\right)-N\left(e^{+} \mu^{-}+e^{-} \mu^{+}\right)$. The background does not contribute to $A_{F}$, so a non-zero value would provide evidence for slepton production.

In the lepton flavor mixing case the pair production of sleptons will produce $e \mu$ events with $\not p_{T}$ - these events will be referred to as "flavor violating" dilepton events. The background to this signal is from the same sources as for the flavor conserving dilepton signal (with the same rate) as well as from $\tilde{\tau} \tilde{\tau}^{*}$ production followed by leptonic decays of $\tau \mathrm{s}$.

The detection of SUSY lepton flavor violation using the above flavor violating dilepton events for the CMS detector at the LHC was studied in references [4, 5] for the case of maximal mixing $(\theta=\pi / 4)$. With the mixing angle being maximal, the flavor violating dilepton signal rate is high; see Eqn.(5) (assuming $x \sim 1$ ). In fact, the number of flavor conserving and flavor violating events from slepton production in this case are the same and each is equal to one half the signal

\footnotetext{
${ }^{7}$ Alignment models with $\Delta m \sim m$ are not considered here since $\sin \theta \sim O\left(10^{-2}\right)$.
} 
in the zero mixing case so that $A_{F} \approx 0$ (unlike the case of zero or non-maximal mixing). In the case where the production cross-sections for staus and the lightest charginos are comparable to that of the sleptons, the production rate for the SUSY background to $e \mu$ events is $\sim 4 \%$ of the total flavor conserving signal (in the absence of mixing). P Thus, the chargino and stau backgrounds are much smaller. The high signal and low SUSY background rate (compared to the signal) for maximal mixing enables detection of a $5 \sigma$ flavor violating signal for sleptons masses up to $250 \mathrm{GeV}$ and LSP masses $m_{\chi_{1}^{0}}<0.4 m_{\tilde{e}_{R}}$ with an integrated luminosity of $100 \mathrm{fb}^{-1}$.

There are some objections to the generality of this result, though. A more general spectrum could result in a larger chargino or stau background. For example, there is no reason to expect the chargino production cross-section to be related to the slepton production cross-section. However, as mentioned above, the kinematics of slepton production and decay are different enough from that of the chargino background that an appropriate set of kinematic cuts could distinguish the two, at least for supergravity motivated parameter choices with comparable squark and gluino masses [7]. Next, the stau background is sensitive to the stau mass, which is likely to differ from the selectron and smuon masses ?. The stau background has softer leptons, so a cut on the $p_{T}$ of the leptons may help distinguish this background from the signal. The success of this may require large statistics and knowledge of the stau production cross-section. Thus, in general, the SUSY background may not be small.

Next, detection of flavor violation for smaller mixing angles is discussed. Since the signal is $\propto \sin ^{2} \theta$, it is significantly smaller for say Cabibbo-like mixing angles. In this case, it is crucial to know the SUSY background more precisely since it is comparable to the signal (assuming similar cross sections for sleptons and charginos). While the quantity $A_{F}$ (>0 for non-maximal mixing) is, up to statistical fluctuations, background-free as far as slepton detection is concerned, it is not useful for providing evidence for slepton flavor violation since the chargino background would need to be determined first. This is because the deviations in the values of $A_{F}$ and $N(e \mu)$ from the SM for a non-zero mixing angle could be reproduced, in the case of zero mixing angle, with a lower slepton production cross-section and a higher chargino production cross-section.

Even if the SUSY background can be reduced sufficiently by an appropriate set of cuts, since the signal is suppressed by the small mixing angle (there will also be a reduction of the signal due to these cuts), it may not possible to probe Cabibbo-like mixing angles. For example, in the case of no mixing, Table 4 of reference [5] gives 195 dilepton signal events for the set of cuts labeled 1 with $L=10 \mathrm{fb}^{-1}$ and a slepton mass of $100 \mathrm{GeV}$. The number of signal events in the case of mixing for $L=100 \mathrm{fb}^{-1}$ is then $1950 \times 2 \times \sin ^{2} \theta \cos ^{2} \theta$ (assuming $x \sim 1$ ). The SM

\footnotetext{
${ }^{8}$ Here, it is assumed that $B R\left(\chi^{+} \rightarrow W^{+} \chi_{1}^{0}\right) \approx 100 \%$ so that the leptonic BRs of $\chi^{+}$are the same as for $W$. If the left-handed sleptons are lighter than $\chi^{+}$, then the leptonic BRs of $\chi^{+}$may be enhanced substantially, in turn increasing the SUSY background.

${ }^{9}$ The rare decays $\tau \rightarrow e \gamma, \tau \rightarrow \mu \gamma$ and $\mu \rightarrow e \gamma$ allow for $O(1)$ splitting between the third and first two generation scalars for $C K M$-like mixing angles.
} 
background from $W W$ production is 9920 for the same set of cuts. Thus a $5 \sigma$ signal (requiring $S / \sqrt{B}>5$ ) is possible only for $\sin \theta \gtrsim 0.4$. Since this signal was obtained for a $24 \mathrm{GeV} \mathrm{LSP}$, only larger angles will be probed for larger LSP masses (since the leptons will be softer in that case). For sleptons heavier than $100 \mathrm{GeV}$ the prospects for detecting small mixing angles are clearly worse.

Thus, in the situation where the SUSY background is known to be small, e.g. if an appropriate set of cuts for a more general spectrum can separate the chargino background from the signal, then the flavor violating dilepton events from Drell-Yan production of sleptons is a promising signal for the detection of flavor violation in the case of large mixing angles. Otherwise, it is important to look for other discovery channels for slepton flavor violation.

\section{Slepton Production in Cascade Decays}

The other way to produce sleptons is through the cascade decays of gluinos and squarks. At the LHC, the production cross sections of squarks and gluinos are much larger than the Drell-Yan production of sleptons, neutralinos, and charginos. So, a larger production of sleptons (if they are light) is expected in the cascade decays than from direct Drell-Yan production. In a generic SUSY event, the production of two real (or virtual from gluino decay) squarks will be followed by their cascade decays ultimately to the LSP through intermediate electroweak sparticles (sleptons, charginos, neutralinos). Assuming for simplicity that the spectrum is gaugino-like, i.e., $\chi_{2}^{0} \approx \tilde{W}_{3}$, $\chi_{1}^{+} \approx \tilde{W}^{+}$and $\chi_{1}^{0} \approx \tilde{B}$, the following squark decays are obtained:

$$
\begin{aligned}
B R\left(\tilde{q}_{R} \rightarrow q \chi_{1}^{0}\right) & \approx 1, \\
B R\left(\tilde{q}_{L} \rightarrow q \chi_{2}^{0}\right) & \approx \frac{1}{3}, \\
B R\left(\tilde{q}_{L} \rightarrow q^{\prime} \chi_{1}^{+,-}\right) & \approx \frac{2}{3} .
\end{aligned}
$$

Thus, a typical SUSY event is:

$$
\begin{aligned}
p p & \rightarrow \tilde{g} \tilde{g}, \tilde{g} \tilde{q} \rightarrow \tilde{q} \tilde{q} \\
& \rightarrow \chi_{E W} \chi_{E W}^{\prime}+X
\end{aligned}
$$

with $\chi_{E W}, \chi_{E W}^{\prime}$ one of $\chi_{1,2}^{0}, \chi_{1}^{+,-}$.

\subsection{Dilepton Events}

If one of the squarks decays to $\chi_{2}^{0}$ followed by the decay of $\chi_{2}^{0}$ to a slepton (if $B R\left(\chi_{2}^{0} \rightarrow \tilde{l} l\right)$ is significant) a large number of $e \mu$ events in the presence of lepton flavor mixing (see Eqns.(6) and (9)) is obtained. These events also have at least 2 high $p_{T}$ jets and large $\not p_{T}$. 
There is no background from $W^{+} W^{-}$production since this background contains no hard jets (assuming jet detection is good). There is a background from $t \bar{t}$ production followed by leptonic decays of the $W$ 's from the top quarks. This can be reduced by rejecting events with $b$-jets or using a high $\not p_{T}$ cut.

There is a SUSY background from the decays of both squarks to charginos, followed by chargino decays to $W^{+}, W^{-}$or $\tilde{l}, \tilde{l}^{*}$. This background is distinguishable from the signal though. The invariant mass distribution of the 2 leptons from the $\chi_{2}^{0}$ decay has a sharp edge (which is a function of the neutralino and slepton masses) [8, 9] unlike the case of the 2 leptons from $\chi^{+} \chi^{-}$ decays. Also, the angle between the 2 leptons from the decay of $\chi_{2}^{0}$ is likely to be smaller than in the case of 2 leptons from $\chi^{+}$and $\chi^{-}$. Such kinematic cuts on the invariant mass of the dileptons and the angle between them easily reduce the number of background events sufficiently if we are interested in detecting flavor conserving dileptons from $\chi_{2}^{0}$ decays.

But, in the case of the flavor violating dilepton events, (as in Section 2) since the signal is suppressed by the mixing angle (while the background is the same), the number of background events that survive (relative to the signal) after cuts depends crucially on the model-dependent cross sections for producing $\chi^{+} \chi^{-}$vs. $\chi_{2}^{0}$ 四. So in general it is difficult to be sure that the cuts have reduced the background sufficiently.

In the circumstance that $\chi^{+} \chi^{-}$are dominantly produced from $\tilde{g} \tilde{g}$ cascade decays, the $\chi^{+} \chi^{-}$ flavor violating background can be estimated as follows. An equal number of like-sign and unlikesign chargino pairs are expected since $\tilde{g}$ is a Majorana particle. The like-sign chargino pairs produce like-sign dileptons so that the opposite-sign chargino $e \mu$ background can be estimated from the number of like-sign $e e$ and $\mu \mu$ events. Unfortunately, in the more general case the $\chi^{+} \chi^{+}$ and $\chi^{+} \chi^{-}$production cross sections are not related since the chargino pairs do not always come from gluino pair decays. For example, $p p \rightarrow \tilde{q}_{L} \tilde{q}_{L}^{*}$ can lead to $\chi^{+} \chi^{-}$, but not to $\chi^{+} \chi^{+}$.

It might be possible to estimate the $\chi^{+} \chi^{-}$background by analysing the (observed) (signal + background) distribution of the invariant mass of the flavor violating dileptons [10]. As mentioned earlier, the dilepton invariant mass distribution for $\chi_{2}^{0}$ decay has a sharp edge unlike the case of the background. The position of this edge (denoted by $M_{l l}$ ) can be easily found by

\footnotetext{
${ }^{10}$ For example, the ratio of the number of events with $\chi^{+} \chi^{-}$to those with (at least) $1 \chi_{2}^{0}$ is larger for $s$-channel $\tilde{q} \tilde{q}^{*}$ production than for gluino pair production which is seen as follows. For the $\tilde{g} \tilde{g}$ case, the probability of getting $2 \tilde{q}_{L}$ is $1 / 4$ compared to a probability of $3 / 4$ for getting at least one $\tilde{q}_{L}$ whereas for $s$-channel $\tilde{q} \tilde{q}^{*}$ production the probabilities are the same. Same sign chargino events are also obtained from $\tilde{g} \tilde{g}$ production whereas $s$-channel $\tilde{q} \tilde{q}^{*}$ production can give only opposite-sign chargino pairs. Thus, if the $s$-channel $\tilde{q} \tilde{q}^{*}$ production is larger, the number of $\chi^{+} \chi^{-}$events relative to $\chi_{2}^{0}$ events increases.

11 There is also a SUSY background from $\chi_{2}^{0}$ decays to $\tilde{\tau} \tau$ followed by leptonic decays of the $\tau$ 's. A cut on the dilepton invariant mass can reduce this: the leptons from the $\tau$ decays are softer than those from the $\tilde{e} / \tilde{\mu} / \chi_{2}^{0}$ decays and so have a smaller invariant mass. But, since, in general, $B R\left(\chi_{2}^{0} \rightarrow \tilde{\tau} \tau\right)$ is not related to $B R\left(\chi_{2}^{0} \rightarrow \tilde{e} e\right)$, as for the chargino background, we cannot be sure that the $\tilde{\tau} \tau$ background has been sufficiently reduced (by the cuts) since this background is unknown.
} 
looking at the distribution of the invariant mass of flavor conserving dileptons (where the $\chi^{+} \chi^{-}$ background is very small) [8, 9]. The existence of an edge in the (observed) opposite-flavor dilepton distribution at $M_{l l}$ would then be indication of flavor violation. However, since the flavor violating dilepton signal is suppressed by (small) mixing relative to the flavor conserving dilepton signal (whereas the $\chi^{+} \chi^{-}$background is the same for both kinds of dileptons), the edge at $M_{l l}$ in the opposite flavor dilepton case might not be as sharp as for the same flavor dilepton case - this depends on the model-dependent cross sections for producing $\chi^{+} \chi^{-}$vs. $\chi_{2}^{0}$.

Next, in the distribution of the invariant mass of the flavor violating dileptons, the events beyond $M_{l l}$ (this value can be obtained from the same flavor dilepton distribution if the edge is not so sharp in the opposite-flavor dilepton distribution) are mostly from the $\chi^{+} \chi^{-}$background [10]. Extrapolating (assuming say a flat distribution for the $\chi^{+} \chi^{-}$background) from the data in this region, the $\chi^{+} \chi^{-}$background in the region with invariant mass less than $M_{l l}$ can be estimated. An excess of $e \mu$ events (with invariant mass between zero and $M_{l l}$ ) over this estimate will be a signal for flavor violation. $\square$ This extrapolation may not be reliable for invariant masses much smaller than $M_{l l}$ since the distribution of the $\chi^{+} \chi^{-}$background in this region is not known. A detailed simulation is required to know this distribution (it is known only that it does not have an edge at $M_{l l}$ ). Near $M_{l l}$ the extrapolation should be more reliable and that is the region where the signal is peaked (since the flavor violating dileptons from $\chi_{2}^{0}$ decay also have a sharp edge at $M_{l l}$ ). An excess in this region (rather than the whole region between zero mass and $M_{l l}$ ) might thus be a better signal for flavor violation [10 - as mentioned earlier, the distribution will have a edge (or a "step") at $M_{l l}$. Also, the $\tilde{\tau} \tau$ background in the region near $M_{l l}$ is negligible since the leptons from these decays are softer [10]. However, statistics are larger if the region from zero mass to $M_{l l}$ is used.

The chargino background can also be eliminated in considering a flavor violating and $C P$ violating dilepton signal [3]. The presence of non-trivial phases in the slepton mixing matrix $W$ breaks $C P$, and results in a non-vanishing asymmetry: $N\left(e^{+} \mu^{-}-e^{-} \mu^{+}\right) \neq 0$. In this case, the $\chi^{+} \chi^{-}$background is not important since it is CP symmetric.

To summarize, if the number of $e \mu$ events (that pass certain cuts) from either Drell-Yan or cascade production is used to detect flavor violation, the SUSY background from $\chi^{+} \chi^{-}$pairs (which passes the same cuts) is difficult to estimate, in general, and may be too large. The possibility of using the observed opposite-flavor dilepton mass distribution (in the case of cascade decays) to estimate the chargino background is interesting, though, and warrants further study [10.

\footnotetext{
${ }^{12}$ The invariant mass of the leptons from the $\tilde{\tau} \tau$ decays (from $\chi_{2}^{0}$ ) is less than $M_{l l}$ and so this background cannot be estimated this way.
} 


\subsection{Events with 4 leptons}

A dramatic flavor violating signal is obtained through the pair production of two $\chi_{2}^{0} \mathrm{~s}$, followed by the decays of both $\chi_{2}^{0}$ s to slepton and lepton pairs. Such an event contains 4 leptons and occurs if both squarks in a SUSY event decay into $\chi_{2}^{0}$. If one of the $\chi_{2}^{0} \mathrm{~s}$ has a flavor violating decay: $\chi_{2}^{0} \rightarrow \tilde{l} l \rightarrow e \mu$, then events containing $3 e 1 \mu$, or $3 \mu 1 e$ will be produced. A typical decay chain is then:

$$
\begin{aligned}
\tilde{q}_{L} \tilde{q}_{L}^{\prime} & \rightarrow \chi_{2}^{0} q+\chi_{2}^{0} q^{\prime} \\
\chi_{2}^{0} & \rightarrow \tilde{l} l \rightarrow e^{+} e^{-} \chi_{1}^{0} \\
\chi_{2}^{0} & \rightarrow \tilde{l}^{\prime} l^{\prime} \rightarrow \mu^{+} e^{-} \chi_{1}^{0} .
\end{aligned}
$$

These events are identified by 4 isolated leptons (with the $3+1$ flavor structure), at least 2 high $p_{T}$ jets, $\not p_{T}$, and concentrating on only those events produced from the decays of first two generation squarks, no $b$-jets. These events will be referred to as "flavor violating" 4 lepton events. The absence of $b$-jets is important in distinguishing the signal from other SUSY and SM backgrounds (see below).

The backgrounds to these events arise from both SM and SUSY sources.

The dominant SM background occurs from $t \bar{t}$ production with semileptonic decays of the $b s$ (or $t \bar{t} \gamma$ production with 2 leptons from $\gamma$ ) and leptonic decays of the $W$ s. In this case, however, the leptons from $b$ decays will not be isolated (or the invariant mass of 2 of the leptons will be zero in the case of $t \bar{t} \gamma$ ). Also, these events have $2 b$ quarks and can be rejected using $b$-jet veto. Double gauge boson production can give 4 lepton events, but none of these events have the $3+1$ flavor structure. Triple gauge boson production ( $W W Z$ or $W W \gamma$ ) can give events with 4 leptons and the correct flavor asymmetry, but some initial state gluon radiation is needed to give the 2 hard jets. The production cross-section for such events is small. Also, events of this kind can also be rejected since the invariant mass of 2 of the leptons will either be zero or $m_{Z}$.

One important obstacle in identifying flavor-violating dilepton events was the potentially large background from $\chi^{+} \chi^{-}$production. In the 4 lepton signal, however, there is no $\chi^{+} \chi^{-}$ background from the squark decays since this gives only 2 leptons.

The weak decay $\tilde{q} \rightarrow W \tilde{q}^{\prime}$, if kinematically allowed, can lead to a possible background. For example, the process

$$
\begin{aligned}
\tilde{q}_{L} \tilde{q}_{L}^{\prime} & \rightarrow W^{-} \tilde{q}_{L}^{\prime \prime}+\chi_{1}^{+} q \\
W^{-} & \rightarrow e^{-} \bar{\nu} \\
\tilde{q}_{L}^{\prime \prime} & \rightarrow \chi_{2}^{0} q^{\prime \prime} \rightarrow \mu^{+} \mu^{-}+\cdots \\
\chi_{1}^{+} & \rightarrow \mu^{+} \nu \chi_{1}^{0}
\end{aligned}
$$

is a potential background. For the first two generation squarks, however, the decay $\tilde{q} \rightarrow W \tilde{q}^{\prime}$ is kinematically forbidden. This is because the mass splitting in an electroweak doublet occurs 
from the electroweak $D$-terms and is less than $m_{W}^{2} / m_{\tilde{q}}<m_{W}$. This process is allowed for the top and bottom squarks, but such an event contains $2 b$-jets and this background can be reduced with a $b$-jet veto.

There is a SUSY background to the flavor violating 4 lepton events from production of heavier neutralinos or chargino in the cascade decays of squarks. For example,

$$
\begin{aligned}
\tilde{q}_{L} \tilde{q} & \rightarrow \chi_{3}^{0} \chi_{2}^{0}+\cdots, \\
\chi_{3}^{0} \rightarrow W^{+} \chi^{-} & \rightarrow e \mu+\cdots, \\
\chi_{2}^{0} \rightarrow \tilde{l l} & \rightarrow e e(\text { or } \mu \mu) \chi_{1}^{0} .
\end{aligned}
$$

This background is small in the so-called gaugino-like region. In this region there is very little gaugino-Higgsino mixing. Then, the heavier chargino and the two heaviest neutralinos are dominantly Higgsinos and the two lightest neutralinos and the lighter chargino are mainly gauginos; this turns out to be typical of the SUSY parameter space still allowed by experimental data. Thus, the decays of the first two generation squarks into the heavier neutralinos or chargino are highly suppressed by the first two generation Yukawa couplings, small gaugino-Higgsino mixing, and also by phase space.

Another potentially large background can also occur from the production of the heavier sleptons (say, the left-handed) and/or sneutrinos. Sleptons can decay to $\chi_{2}^{0} l$ and $\tilde{\nu}$ to $\chi_{1}^{ \pm} l$ if kinematically allowed. If the neutralino and chargino decay to leptons, then this decay chain can give 3 (or 2) leptons. With 1 (or 2) leptons from another decay of this kind (or some other decay chain), this can mimic the flavor violating 4-lepton signal. If the left-handed sleptons are paired produced through the Drell-Yan mechanism, then these events do not contain any hard jets and may be rejected. Thus, the only source for a background from heavier sleptons is their production in the decays of gluinos and squarks. Such a decay does not occur directly, but only through the decays of gluinos and squarks to the heavier neutralino and chargino. The heavier neutralinos and chargino can then decay to the left-handed sleptons. As argued in the previous paragraph though, in the gaugino-like region, the heavier neutralinos/chargino are dominantly Higgsinos so that their decays to the sleptons are suppressed by the lepton Yukawa couplings and small gaugino/Higgsino mass mixing angles. So this background is negligible.

However, top squarks (and bottom squarks for large $\tan \beta$ ) will have significant decay branching fractions into heavier neutralinos or chargino even if they are purely Higgsinos since the third generation Yukawa couplings (and hence couplings of the squarks to Higgsinos) are large. Further, as mentioned earlier, $W$ s may be produced in the direct decay of stops or sbottoms. Also, top quarks from stop or sbottom decays produce $W$ s. Both of these processes give additional isolated leptons. This leads to a potential background even if stops or sbottoms decay only to the lighter chargino and neutralinos. For example, the following decay chain is a possible 
background:

$$
\begin{aligned}
\tilde{t \tilde{t}^{*}} & \rightarrow b \chi^{-}+t \chi_{2}^{0}, \\
t & \rightarrow W^{+} b \rightarrow e^{+} b+\cdots, \\
\chi^{-} & \rightarrow W^{-} \chi_{1}^{0} \rightarrow \mu^{-}+\cdots, \\
\chi_{2}^{0} & \rightarrow e^{+} e^{-} \chi_{1}^{0} .
\end{aligned}
$$

These backgrounds to flavor violating 4 lepton events can be reduced by rejecting any 4 lepton event that contains at least one $1 b$-jet. Note that the top or bottom squark background has at least $2 b$ quarks. The efficiency for rejecting this background is discussed in a later section where a specific spectrum is considered.

There is also an important SUSY background from decays of taus and staus produced from the decays of two $\chi_{2}^{0}$ s. That is,

$$
\begin{aligned}
& \chi_{2}^{0} \rightarrow \tilde{\tau} \tau \rightarrow e \mu \chi_{1}^{0}+\ldots, \\
& \chi_{2}^{0} \rightarrow \tilde{l} l \rightarrow e e(\text { or } \mu \mu) \chi_{1}^{0} .
\end{aligned}
$$

This background can be estimated/measured as follows. In the above decay chain, if one $\tau$ decays hadronically instead of leptonically, the result is $3 e 1 \tau$-jet events. If a lower bound on the $\tau$-jet detection efficiency is known, an upper bound on the number of $3 e 1 \mu$ events coming from $\tau$ decays is obtained by using the number of $3 e 1 \tau$-jet events. An excess of $3 e 1 \mu$ events over this background is a signature of lepton flavor violation.

Lastly, the following $\chi_{2}^{0}$ decay chains can also give flavor violating dileptons:

$$
\begin{aligned}
\chi_{2}^{0} & \rightarrow h(\text { or }) Z \chi_{1}^{0} \\
h(\text { or }) Z & \rightarrow \tau \tau \rightarrow e \mu .
\end{aligned}
$$

In combination with another $\chi_{2}^{0}$ decay to $e e$ or $\mu \mu$, these decay chains can give flavor violating 4-lepton events. In the gaugino region, the decay $\chi_{2}^{0} \rightarrow Z \chi_{1}^{0}$ is suppressed since there is no vertex with $Z$ and 2 neutral gauginos. In any case, an effective $B R\left(\chi_{2}^{0} \rightarrow \tau \tau\right)$ can be defined to include these two decay chains in addition to the $\chi_{2}^{0} \rightarrow \tilde{\tau} \tau$ decay. It will be shown in section 3.2 .2 that this (in general unknown) BR does not affect the estimate of the (effective) $\tau$ background obtained by using the $3 e 1 \tau$-jet events.

\subsubsection{A quick estimate of number of 4 lepton events}

A typical value for the total SUSY production cross section (gluinos and squarks) at the LHC is:

$$
\sigma_{S U S Y} \sim 10 \frac{\pi \alpha_{S}^{2}}{\hat{s}} \sim 100 \mathrm{pb}
$$


with $\sqrt{\hat{s}} \sim 1 \mathrm{TeV}, \alpha_{S} \sim 0.1$ and summed over colors and generations (the factor of 10). Assuming that the probability to get a $\tilde{q}_{L}$ is $1 / 2$ and $B R\left(\tilde{q}_{L} \rightarrow \chi_{2}^{0} q\right)=1 / 3$, this gives

$$
\sigma_{\chi_{2}^{0} \chi_{2}^{0}} \sim \sigma_{S U S Y}\left(\frac{1}{2}\right)^{2}\left(\frac{1}{3}\right)^{2} \sim 3 \mathrm{pb}
$$

If $B R\left(\chi_{2}^{0} \rightarrow \chi_{1}^{0} l^{+} l^{-}\right) \sim 0.16$ (for each of $\left.l=e, \mu\right)$ and for $\sim$ one year of running at low luminosity which gives an integrated luminosity of $L \sim 10(\mathrm{fb})^{-1}$, the expected number of events is :

$$
\begin{aligned}
& N\left(\chi_{2}^{0} \chi_{2}^{0}\right) \sim 30,000, \\
& N(4 l \text { where } l=e, \mu)=(2 \times 0.16)^{2} N\left(\chi_{2}^{0} \chi_{2}^{0}\right) \sim 3300, \\
& N\left(3 l+l^{\prime}\right)=4 \sin ^{2} \theta \cos ^{2} \theta x N(4 l) \sim 550,
\end{aligned}
$$

for $\sin \theta \approx 0.2$ and $x \sim 1$. To be clear,

$$
S_{F V} \equiv N\left(3 l+l^{\prime}\right)=\left(N\left(e^{+} \mu^{-} \mu^{+} \mu^{-}\right)+(+\leftrightarrow-)\right)+(\mu \leftrightarrow e)
$$

and

$$
N(4 l)=N\left(e^{+} e^{-} e^{+} e^{-}\right)+N\left(e^{+} e^{-} \mu^{+} \mu^{-}\right)+N\left(\mu^{+} \mu^{-} \mu^{+} \mu^{-}\right)+S_{F V} .
$$

In the next section this definition for $N(4 l)$ is trivially extended to include leptons produced by the decay of $\tau$ s. Thus, typically, a large number of 4 lepton flavor violating events is expected from the cascade decays of squarks 10 .

\subsubsection{Detailed estimates at Point 5 (Point $A$ ) of LHC studies}

One Point of the LHC supersymmetry studies [8, 9] contains a spectrum that is favorable for the detection of a flavor-violating 4 lepton signal. The minimal supergravity input parameters for this point are:

$$
\begin{aligned}
& m_{0}=100 \mathrm{GeV}, \quad M_{1 / 2}=300 \mathrm{GeV}, \quad A_{0}=300 \mathrm{GeV} \text {, } \\
& \tan \beta=2.1, \quad \operatorname{sgn}(\mu)=+, \quad m_{\text {top }}=170 \mathrm{GeV} .
\end{aligned}
$$

Renormalization group evolution of these input parameters to the weak scale results in a mass spectrum which is given in Table 1. Note that $m_{\chi_{2}^{0}} \approx 230 \mathrm{GeV}>m_{\tilde{l}_{R}} \approx 160 \mathrm{GeV}$ so that the decay of $\chi_{2}^{0}$ into real sleptons is allowed.

The production cross-section for SUSY particles is presented in Table 2, and is dominated by $\tilde{g} \tilde{q}$ production. In total $\sigma_{S U S Y} \approx 16 \mathrm{pb}$. To estimate the number of signal and background

\footnotetext{
${ }^{13}$ Both $\chi_{2}^{0}$ s decaying to flavor violating dileptons gives $\left(e^{+} \mu^{-}\right)\left(e^{+} \mu^{-}\right)$and $\left(e^{+} \mu^{-}\right)\left(\mu^{+} e^{-}\right)$events. The latter cannot be distinguished from the events where one $\chi_{2}^{0}$ decays to $e^{+} e^{-}$and the other to $\mu^{+} \mu^{-}$. The former events can also be used as a signal of flavor violation, but the number of these events is expected to be very small since they require both $\chi_{2}^{0}$ s to decay into flavor violating dileptons. For simplicity these events were not included in Eqn.(23).
} 


\begin{tabular}{llllllll}
\hline$\tilde{g}$ & 770 & $\tilde{q}_{L}$ & 685 & $\tilde{q}_{R}$ & 660 & $h$ & 100 \\
\hline$\tilde{t}_{1}$ & 500 & $\tilde{t}_{2}$ & 715 & $\tilde{b}_{1}$ & 635 & $\tilde{b}_{2}$ & 660 \\
\hline$\tilde{l}_{L}$ & 240 & $\tilde{l}_{R}$ & 160 & $\chi_{1}^{ \pm}$ & 230 & $\chi_{2}^{ \pm}$ & 500 \\
\hline$\chi_{1}^{0}$ & 120 & $\chi_{2}^{0}$ & 230 & $\chi_{3}^{0}$ & 480 & $\chi_{4}^{0}$ & 505 \\
\hline
\end{tabular}

Table 1: Mass spectrum in $\mathrm{GeV}$ at LHC Point [8, 9]. Here $\tilde{q}=\tilde{u}, \tilde{d}, \tilde{c}, \tilde{s}$, and $\tilde{l}=\tilde{e}, \tilde{\mu}, \tilde{\tau}$.

\begin{tabular}{cccccc}
\hline$\tilde{g} \tilde{g}$ & 1750 & $\tilde{g} \tilde{q}, \tilde{g} \tilde{q}^{*}$ & 8300 & $\tilde{q} \tilde{q}^{*}$ & 2380 \\
\hline$\tilde{q} \tilde{q}^{\prime}$ & 2820 & $\tilde{b} \tilde{b}^{*}$ & 300 & $\tilde{t} \tilde{t}^{*}$ & 700 \\
\hline
\end{tabular}

Table 2: The production cross-sections in fb for different SUSY particles at the LHC Point [8, 9]. Here all flavors $\tilde{q}_{H}=\tilde{u}, \tilde{d}, \tilde{c}, \tilde{s}$ and $H=L, R$ are summed over.

events, the branching fractions of the sparticles are needed. These are given in Table 3. Note that at this Point $B R\left(\chi_{2}^{0} \rightarrow \tilde{l}_{i} l_{i}\right) \sim 0.12$ and is reduced due to the large branching fraction $B R\left(\chi_{2}^{0} \rightarrow h \chi_{1}^{0}\right)$. This gives from decays of first two generation squarks the number of $\chi_{2}^{0}$ pairs produced:

$$
N\left(\chi_{2}^{0} \chi_{2}^{0}\right)=(0.32)^{2} \times\left(\sigma_{\tilde{g} \tilde{g}} \times(0.3)^{2}+\frac{1}{2} \sigma_{\tilde{g} \tilde{q}} \times 0.3+\frac{1}{4} \sigma_{\tilde{q} \tilde{q}^{\prime}}+\frac{1}{2} \sigma_{\tilde{q}^{*} \tilde{q}}\right) L \approx 3400 .
$$

(The factors of $1 / 2$ and $1 / 4$ are easy to understand: $1 / 2$ of all $\tilde{q} \tilde{q}^{*}$ produced from s-channel gluon and 4-point contact interaction 四, and 1/4 of all $\tilde{q} \tilde{q}^{\prime}$ s produced (from t-channel gluino exchange) are left-handed pairs.) This is for one year of running at low luminosity $\left(L=10 \mathrm{fb}^{-1}\right)$ and for one detector. Hereafter estimates of event numbers will use this integrated luminosity. A realistic detection efficiency of $90 \%$ for single $e, \mu$, and $90 \%$ for the single-prong decay $\tau \rightarrow \pi \nu$ (BR 0.11 ) will be used. These are needed to determine the number of 4-lepton and 3-lepton $+\tau$-jet events that are detected. Later, a comment on a more realistic $\tau$-jet detection will be made.

Next the 4-lepton signal and background are estimated.

Due to the decay chain

$$
\begin{aligned}
\chi_{2}^{0} & \rightarrow h \chi_{1}^{0} \\
h & \rightarrow \tau \tau
\end{aligned}
$$

the effective $B R\left(\chi_{2}^{0} \rightarrow \tau \tau \chi_{1}^{0}\right) \equiv R_{\tau}$ is

$$
\begin{aligned}
R_{\tau} & =B R\left(\chi_{2}^{0} \rightarrow \tilde{\tau}_{R} \tau\right)+B R\left(\chi_{2}^{0} \rightarrow h \chi_{1}^{0}\right) \times B R(h \rightarrow \tau \tau) \\
& =0.15 .
\end{aligned}
$$

\footnotetext{
${ }^{14} \mathrm{It}$ is assumed that all of the $\tilde{q} \tilde{q}^{*}$ production is by this channel. This is reasonable since most of the hard collisions at LHC energies are likely to be gluon-gluon.
} 


\begin{tabular}{cccccc}
\hline$\tilde{g} \rightarrow q \tilde{q}_{L}$ & 30 & $\tilde{g} \rightarrow q \tilde{q}_{R}$ & 30 & $\tilde{g} \rightarrow \tilde{t}_{1} t$ & 14 \\
\hline$\tilde{g} \rightarrow \tilde{b}_{L} b$ & 15 & $\tilde{g} \rightarrow \tilde{b}_{R} b$ & 10 & $\tilde{t}_{2} \rightarrow Z_{0} \tilde{t}_{1}$ & 26 \\
\hline$\tilde{t}_{2} \rightarrow \chi_{4}^{0} t$ & 21 & $\tilde{t}_{2} \rightarrow \chi_{2}^{ \pm} b$ & 18 & $\tilde{t}_{2}\left(\tilde{t}_{1}\right) \rightarrow \chi_{1}^{ \pm} b$ & $15(63)$ \\
\hline$\tilde{t}_{2}\left(\tilde{t}_{1}\right) \rightarrow \chi_{2}^{0} t$ & $8(17)$ & $\tilde{t}_{2} \rightarrow \chi_{3}^{0} t$ & 6 & $\tilde{t}_{2}\left(\tilde{t}_{1}\right) \rightarrow \chi_{1}^{0} t$ & $6(20)$ \\
\hline$\tilde{q}_{L} \rightarrow q \chi_{2}^{0}$ & 32 & $\tilde{q}_{L} \rightarrow q \chi_{1}^{ \pm}$ & 64 & $\tilde{q}_{L} \rightarrow q \chi_{1}^{0}$ & 1.5 \\
\hline$\tilde{q}_{L} \rightarrow q \chi_{2}^{ \pm}$ & 1.5 & $\tilde{q}_{L} \rightarrow q \chi_{4}^{0}$ & 1 & $\tilde{q}_{R} \rightarrow q \chi_{1}^{0}$ & 99 \\
\hline$\chi_{2}^{0} \rightarrow \tilde{l}_{R} l$ & 36 & $\chi_{2}^{0} \rightarrow h \chi_{1}^{0}$ & 63 & $\tilde{l}_{R} \rightarrow l \chi_{1}^{0}$ & 100 \\
\hline$\tilde{l}_{L} \rightarrow \chi_{1}^{0} e$ & 90 & $\chi_{1}^{+} \rightarrow W^{+} \chi_{1}^{0}$ & 98 & $h \rightarrow \tau \tau$ & 5 \\
\hline
\end{tabular}

Table 3: Branching fractions (in percent) for sparticles at LHC Point [9]. Here $\tilde{q}=\tilde{u}, \tilde{d}, \tilde{c}, \tilde{s}$, and $\tilde{l}=\tilde{e}, \tilde{\mu}, \tilde{\tau}$.

Using the above $\mathrm{BR}$ and $B R\left(\chi_{2}^{0} \rightarrow \tilde{l}_{R} l\right)=0.12$ for each of $l=e, \mu$ and $B R(\tau \rightarrow e \nu) \approx$ $B R(\tau \rightarrow \mu \nu) \approx 1 / 2 \times 0.35$,

$$
\begin{aligned}
B R\left(\chi_{2}^{0} \rightarrow e e \chi_{1}^{0}, \mu \mu \chi_{1}^{0}\right)= & 2 \times 0.12 \times\left(1-2 \sin ^{2} \theta \cos ^{2} \theta x\right) \\
& +R_{\tau} \times(0.35)^{2} \times \frac{1}{2}, \\
B R\left(\chi_{2}^{0} \rightarrow e \mu \chi_{1}^{0}\right)= & 2 \times 0.12 \times 2 \sin ^{2} \theta \cos ^{2} \theta x+R_{\tau} \times(0.35)^{2} \times \frac{1}{2},
\end{aligned}
$$

where the first terms in each equation are from decays of $\tilde{e}$ and $\tilde{\mu}$ and the second terms are from $\tau$ decays.

Then, the total number of 4-lepton events expected from $\chi_{2}^{0}$ pair decays (including detection efficiencies, but parameterizing the acceptance cut as $\varepsilon_{C U T}$ - see later 町) is

$$
\begin{aligned}
N(4 l) & =N\left(\chi_{2}^{0} \chi_{2}^{0}\right) \times\left(B R\left(\chi_{2}^{0} \rightarrow e e, \mu \mu, e \mu\right)\right)^{2}(0.9)^{4} \times \varepsilon_{C U T} \\
& =3400 \times\left(0.24+R_{\tau} \times(0.35)^{2}\right)^{2} \times(0.9)^{4} \times \varepsilon_{C U T} \\
& \approx 149 \times \varepsilon_{C U T} .
\end{aligned}
$$

To get $3 e 1 \mu+3 \mu 1 e$ events, one $\chi_{2}^{0}$ has to decay into $e e / \mu \mu$ and the other to $e \mu$. Thus, the number of $3 e 1 \mu+3 \mu 1 e$ events from flavor-mixing for $\sin \theta=0.2$ and $x \sim 1$ (it is shown later that these values are consistent with the $\mu \rightarrow e \gamma$ limit) is

$$
\begin{aligned}
S_{F V}= & N\left(\chi_{2}^{0} \chi_{2}^{0}\right) \times B R\left(\chi_{2}^{0} \rightarrow e e \chi_{1}^{0}, \mu \mu \chi_{1}^{0}\right) \times(0.9)^{4} \times \varepsilon_{C U T} \\
& \times 2 \times 0.24 \times 2 \sin ^{2} \theta \cos ^{2} \theta x \\
\approx & 20 \times \varepsilon_{C U T} .
\end{aligned}
$$

\footnotetext{
${ }^{15}$ Detection efficiency refers to the probability that the lepton (or $\tau$-jet in a later case) will be detected given that it passes the acceptance cuts.
} 
There is an extra factor of 2 since either $\chi_{2}^{0}$ can decay to flavor violating dileptons. Next, the number of $3 e 1 \mu+3 \mu 1 e$ events from leptonic decays of $\tau$ s produced from $\chi_{2}^{0}$ is

$$
\begin{aligned}
B_{F V}= & N\left(\chi_{2}^{0} \chi_{2}^{0}\right) \times 2 \times\left(R_{\tau} \times(0.35)^{2} \times \frac{1}{2}\right) \\
& \times B R\left(\chi_{2}^{0} \rightarrow e e \chi_{1}^{0}, \mu \mu \chi_{1}^{0}\right) \times(0.9)^{4} \times \varepsilon_{4 l} \times \varepsilon_{C U T} \\
\approx & 9 \times \varepsilon_{C U T} .
\end{aligned}
$$

Here, $\varepsilon_{4 l}$ is the acceptance for 4 leptons with 2 of them coming from the decay chain $\chi_{2}^{0} \rightarrow \tau \tau$ relative to that for all 4 leptons coming from $\chi_{2}^{0} \rightarrow \tilde{e} e$ or $\mu \tilde{\mu}$. Since the leptons from the $\tau$ decay are softer, it is expected that $\varepsilon_{4 l} \lesssim 1$. T- To get the number in the last line above, $\varepsilon_{4 l} \approx 1$ has been assumed.

Finally, the above 2 numbers are from the leptonic decays of $2 \chi_{2}^{0} \mathrm{~s}$ from the decays of first two generation squarks only. As mentioned before, stop/sbottom decays to $W$, $\chi_{3}^{0}$ etc. can give a background to the flavor violating 4-lepton signal (see Eqn.(16)). To reject these events, a $b$-jet veto is used. This implies that events with 4 leptons coming from $2 \chi_{2}^{0}$ decays with (at least) one $\chi_{2}^{0}$ coming from a stop/sbottom decay will also be rejected; this is the reason for not including the $\chi_{2}^{0}$ pairs from stop/sbottom decays in the numbers above.

Measuring the background from $\chi_{2}^{0} \rightarrow \tau \tau$ decays is discussed next.

As mentioned earlier, the idea is to measure the number of $(3 e \tau-$ jet $)+(2 e 1 \mu \tau-$ jet $)+\cdots$ events where $\tau$-jet refers to the hadronic decay of $\tau$. At this LHC Point the number of these events (including detection efficiencies) is

$$
\begin{aligned}
N(3 l+\tau-\text { jet })= & N\left(\chi_{2}^{0} \chi_{2}^{0}\right) \times 2 \times\left(R_{\tau} \times 2 \times 0.35 \times \varepsilon_{\tau}\right) \times \varepsilon_{C U T} \times \\
& B R\left(\chi_{2}^{0} \rightarrow e e \chi_{1}^{0}, \mu \mu \chi_{1}^{0}, e \mu \chi_{1}^{0}\right) \times(0.9)^{3} \times \varepsilon_{3 l} .
\end{aligned}
$$

A factor of 2 is due to either $\tau$ decaying to a jet. Here, $\varepsilon_{\tau}$ includes $B R(\tau \rightarrow$ hadron $)$ and the efficiency for detecting a hadronic decay of $\tau$. The variable $\varepsilon_{3 l}$ is the acceptance for $(3+1 \tau-$ jet $)$ relative to that for 4 leptons all of which come from the decay chain $\chi_{2}^{0} \rightarrow \tilde{e} e, \tilde{\mu} \mu$. It is expected that $1 \gtrsim \varepsilon_{3 l} \gtrsim \varepsilon_{4 l}$ since the lepton from the $\tau$ decay is softer than the $\tau$-jet and since the $\tau$ decay products (both lepton and jet) are softer than the leptons from the decay chain $\chi_{2}^{0} \rightarrow \tilde{e} e, \tilde{\mu} \mu$. From Eqns.(31) and (32) and assuming $B R\left(\chi_{2}^{0} \rightarrow e e \chi_{1}^{0}, \mu \mu \chi_{1}^{0}\right) \approx B R\left(\chi_{2}^{0} \rightarrow e e \chi_{1}^{0}, \mu \mu \chi_{1}^{0}, e \mu \chi_{1}^{0}\right)$, the following relation is obtained

$$
B_{F V} \approx N(3 l+\tau-\text { jet }) \times \frac{0.9 \times \frac{0.35}{2} \times \varepsilon_{4 l}}{2 \times \varepsilon_{3 l} \times \varepsilon_{\tau}} .
$$

Note that $R_{\tau}$ cancels in the ratio. Thus, using the $(3 l+\tau-$ jet $)$ detection together with an understanding of the $\tau$ detection efficiency $\left(\varepsilon_{\tau}\right)$, as well as the acceptance for 4 leptons (with 2

\footnotetext{
${ }^{16}$ Strictly speaking, the factor $\varepsilon_{4 l}$ should be included in determining $N(4 l)$ and $S_{F V}$ as well. But since the number of events in these samples from $\tau$ decays is very small, it is a good approximation to assume $\varepsilon_{4 l} \approx 1$ in those numbers.
} 
of them from $\tau$ decays) versus (3 leptons $+\tau$-jet) $\left(\varepsilon_{4 l} / \varepsilon_{3 l}\right)$, the number of $(3 e 1 \mu+3 \mu 1 e)$ events from $\tau$ decay (Eqn.(31)) contained in the full 4-lepton sample can be obtained from the above relation. This is important, as it means that the $\chi_{2}^{0} \rightarrow \tau \tau$ background to the flavor-violating signal can be determined without knowing the relative branching fraction of $\chi_{2}^{0}$ to $h, \tilde{l} l$, or $\tilde{\tau} \tau$.

Assuming that the detection efficiency for the decay $\tau \rightarrow \pi \nu$ (which has a BR of 0.11 ) is 0.9 so that $\varepsilon_{\tau} \approx 0.9 \times 0.11$, and assuming $\varepsilon_{3 l} \approx 1$ gives

$$
N(3 l+\tau-\text { jet }) \approx 11 \times \varepsilon_{C U T} .
$$

Independent of this, it is worth remarking that with enough stastistics it might be possible to measure $B R\left(\chi_{2}^{0} \rightarrow h \chi_{1}^{0}\right), B R\left(\chi_{2}^{0} \rightarrow \tilde{e} e, \tilde{\mu} \mu\right)$ and $B R\left(\chi_{2}^{0} \rightarrow \tilde{\tau} \tau\right)$ assuming that these are the dominant decay modes of $\chi_{2}^{0}$. The decay chain $\chi_{2}^{0} \rightarrow h \chi_{1}^{0} \rightarrow b \bar{b} \chi_{1}^{0}$ (where $\chi_{2}^{0}$ is from cascade decays of squarks as usual) gives $b \bar{b}$ events with high $p_{T}$ jets and $\not_{T}$. Comparing these to the number of dilepton events from $\chi_{2}^{0}$ decays gives

$$
\frac{N(b \bar{b})}{N(2 l)} \propto \frac{B R\left(\chi_{2}^{0} \rightarrow h \chi_{1}^{0}\right)}{B R\left(\chi_{2}^{0} \rightarrow \tilde{e} e \chi_{1}^{0}, \tilde{\mu} \mu \chi_{1}^{0}\right)} .
$$

Similarly, the number of $3 l+1 \tau$-jet events compared to 4 -lepton events is

$$
\frac{N(3 l+\tau-\text { jet })}{N(4 l)} \propto \frac{R_{\tau}}{B R\left(\chi_{2}^{0} \rightarrow \tilde{e} e \chi_{1}^{0}, \tilde{\mu} \mu \chi_{1}^{0}\right)} .
$$

All the events in the above two equations have in addition high $p_{T}$ jets and $\not_{T}$ to make sure that these are from cascade decays of squarks. From these two measurements and the assumption that $\sum B R s=1$ the above-mentioned branching ratios can be obtained. This could provide complementary information to the flavor violating signal discussed here.

Returning to the main subject of this section, an observation of an excess of the 'flavor violating' 4-leptons events over those from $\tau$ decay (Eqn.(31)) would be a strong evidence for lepton flavor violation. But, before concluding that SUSY lepton flavor violation has been detected, the background to the flavor violating 4 lepton events from stop/sbottom production (see Eqn.(37) below) must be removed, and also the $\tau$-jet detection efficiency $\varepsilon_{\tau}$ must be known. These two issues are discussed next.

The $\tau$ hadronic decays from $Z \rightarrow \tau \tau$ at the LHC were simulated for the ATLAS detector in [11] [7. This study shows that a detection efficiency $\epsilon_{\tau}$ for a hadronic $\tau$ decay (including the multi-prong decays, i.e, a total $\tau$ decay $\mathrm{BR}$ of 0.65$)$ of $\approx 40 \times 0.65 \%$ with a rejection factor of 15 for non- $\tau$ jets can be achieved. This is possible since $\tau$-jets have lower particle multiplicity, narrower profile and smaller invariant mass than the QCD jets [11]. A similar detection efficiency (or even better detection efficiency and rejection of non- $\tau$ jets if the strategy is optimized for this case) for $\tau$-jets from sparticle decays could be expected.

\footnotetext{
${ }^{17}$ There is also a study of detecting $\tau$-jets from heavy SUSY Higgs decay for the CMS detector [12].
} 
The important point about this though is that it suffices to know a lower limit on the $\tau$-jet detection efficiency to get an upper limit on the number of $(3 e 1 \mu)+(3 \mu 1 e)$ events from tau decays using the (3e $\tau$ - jet) events (see Eqn.(33)). Similarly, since $\varepsilon_{4 l} \lesssim \varepsilon_{3 l}$, an upper limit on $B_{F V}$ can be obtained even though these $\varepsilon$ 's may not be known precisely. Also, if the $\tau$-jet detection (and QCD jet rejection) is good, there will be large number of events with 2 leptons and $2 \tau$-jets from $2 \chi_{2}^{0}$ decays. These can be used in addition to the 3 lepton $1 \tau$-jet events to estimate the background to flavor violating 4 lepton events from $\tilde{\tau} / \tau$ decays.

To reduce the stop and sbottom backgrounds a $b$-jet veto can be used. Before using this veto, the number of expected $3 e 1 \mu+3 \mu 1 e$ events from decays of $\tilde{t}$ or $\tilde{b}$ to $W, \chi_{3}^{0}, \chi_{1}^{ \pm}$etc. (in the absence of any flavor mixing) can be estimated using the production cross-sections and branching fractions from Tables 2 and 3 . The result is, including lepton detection efficiencies:

$$
N(\tilde{t} \text { or } \tilde{b}) \approx 50 \times \varepsilon_{C U T}
$$

Each of these events has at least $2 b$ quarks. So with a $b$-detection efficiency of $60 \%$ (and rejection factor of 200 against non- $b$ jets at low luminosity [13]), the number of $3 e 1 \mu+\cdots$ events from stop/sbottom decays after the $b$-jet veto goes down to 8 . This can be further reduced by using a $b$-tagging efficiency of $90 \%$ with a mistag rate of $25 \%$ (i.e., rejection factor of 4 against non- $b$ jets) at low luminosity [9, 13]; this will reduce the signal by a bit. This strategy can be optimized depending on the luminosity [13].

Lastly, to get actual number of events, the cuts used to select these events must also be taken into account. The effect of these cuts on the signal and background rates is buried in the fudge factor $\varepsilon_{C U T}$. For example, $p_{T} \gtrsim 10 \mathrm{GeV}$ and $|\eta| \lesssim 2.5$ is required to be able to detect $e$ or $\mu$. Also, to reduce any remaining small SM background, i.e., to make sure that these are SUSY events, various cuts on $\not p_{T}, p_{T}$ of jets, a variable $M_{\text {eff }}$ [8, 9] related to $\not p_{T}, p_{T}$ of jets, can be imposed. Analysis of the events simulated in [9] showed that there were $\sim 40$ events with 4 leptons with no $b$-jets that pass all the cuts mentioned above compared to the estimate of $\sim 149$ from cross sections and BRs, Eqn.(29): there is an acceptance factor of $\varepsilon_{C U T} \sim 1 / 4$ from the various kinematic cuts. We have also checked that almost all of these (simulated) events have 2 $\chi_{2}^{0} \mathrm{~s}$ as expected. 18 There are very few events in this sample (from the simulation) with heavier neutralinos/chargino in agreement with the expectation from the very small BRs of the first two generation squarks to these sparticles at this point in the SUSY parameter space [9] (see Table 3). The number of events (from the simulation) with at least $1 b$ quark and 4 leptons is also in rough agreement (up to the acceptance factor) with the number of 4 lepton events with at least 1 stop/sbottom expected from the cross section and branching fraction estimates. 19

\footnotetext{
${ }^{18}$ The information about whether an event in the simulation has $\chi_{2}^{0} \mathrm{~s}, \tilde{t} \mathrm{~s}, \chi_{3}^{0} \mathrm{~s}$ etc. is from the event generator.

${ }^{19} \mathrm{We}$ have also checked that these simulated events $d o$ have at least 1 stop/sbottom. There are very few events in this sample with no stops/sbottoms but with $b$-jets from initial state gluon radiation.
} 
Including an acceptance factor of $\varepsilon_{C U T} \sim 1 / 4$ for both background and signal, a $b$-jet detection efficiency of $60 \%$ (which was not included in Eqn.(37)) and detection efficiency of $90 \%$ for the decay $\tau \rightarrow \pi \nu$, and a 66\% 4-lepton detection efficiency (the $\tau$ and lepton detection efficiencies were included in the previous estimates of $S_{F V}$ etc.), a summary of the expected number of events at low luminosity is :

$$
\begin{aligned}
N(4 l) & \approx 37 \times \frac{L}{10 \mathrm{fb}^{-1}} \\
S_{F V} & \approx 5 \times \frac{\sin ^{2} 2 \theta}{0.15} x \times \frac{L}{10 \mathrm{fb}^{-1}} \\
B_{F V} & \approx 2 \times \frac{L}{10 \mathrm{fb}^{-1}} \\
N(3 l+\tau-\text { jet }) & \approx 3 \times \frac{L}{10 \mathrm{fb}^{-1}} \\
N(\tilde{t} \text { or } \tilde{b}) & \approx 2 \times \frac{L}{10 \mathrm{fb}^{-1}} .
\end{aligned}
$$

While these numbers may be a little small for one detector and one year of running at low luminosity $\left(L=10 \mathrm{fb}^{-1}\right)$, there is cause for optimism. More integrated luminosity $L$ from $>1$ year of running and/or 2 detectors can significantly increase the statistics. Further, a larger $B R\left(\chi_{2}^{0} \rightarrow \tilde{l} l\right)$ would give more statistics. This could occur at a point in the SUSY parameter space with a heavier Higgs boson, and thus a lower $B R\left(\chi_{2}^{0} \rightarrow h \chi_{1}^{0}\right)$.

To illustrate the discovery or exlcusion significance of these results, an integrated luminosity of $L=100 \mathrm{fb}^{-1}$ is considered. This could occur for 5 years of running at low luminosity for two detectors 0 . For this integrated luminosity there are 22 4-lepton flavor violating events from the $\tilde{\tau} / \tau$ background, and 30 3-lepton $\tau$-jet events. There will also be 1254 -lepton flavor violating events before the $b$-jet veto from the $\tilde{t} / \tilde{b}$ background. Next, the $b$-tagging efficiency is optimized so that the $\tilde{t} / \tilde{b}$ background is (less than or) equal to the $1 \sigma$ statistical error in $\tilde{\tau} / \tau$ background while at the same time the reduction of the signal due to mistagging is small. This is achieved with a $b$-tagging efficiency of $80 \%$, rather than the $60 \%$ of before. At this higher tagging efficiency there is a mistag rate of 1 in 50 , so there is very little reduction of the signal. With an $80 \% b$-tagging efficiency, $5 \tilde{t} / \tilde{b}$ background events remain since each event has at least $2 b$-jets. Then the background is dominated by the $\tilde{\tau} / \tau$ decays. A $5 \sigma(2 \sigma)$ discovery (exclusion) requires that $S / \sqrt{B}>5(S / \sqrt{B}>2)$, and this requires $>23(>9)$ signal events. So a $5 \sigma$ discovery is obtained for

$$
\sqrt{x} \sin 2 \theta_{R}>0.26(5 \sigma \text { discovery }) \text { or } \sin \theta_{R}>0.13 \text { for } x \sim 1 .
$$

\footnotetext{
${ }^{20}$ One year of running at high luminosity is also possible. In this case however, the $b$-jet mistag rate increases to 1 in 6 for a $b$-tagging efficiency of $80 \%$ [13]. Since most of the signal events occur from $\tilde{q} \tilde{g}$ production and so contain at least three hard jets, approximately $40 \%$ of the signal could be rejected. In this case the discovery (and exclusion) limits on $\sin \theta_{R}$ increase by about $25 \%$. In addition, the tau-jet detection efficiency at high luminosity is not known since a low luminosity was used in the ATLAS study.
} 
If no signal is observed then the $2 \sigma$ exclusion limit is

$$
\sqrt{x} \sin 2 \theta_{R}>0.16(2 \sigma \text { exclusion }) \text { or } \sin \theta_{R}>0.08 \text { for } x \sim 1 \text {. }
$$

To end this Section, these values of $\sin 2 \theta_{R}$ and $\Delta m / m$ that may be probed by the LHC are compared to the constraints on these parameters obtained from $\mu \rightarrow e \gamma$. The LHC signal is proportional to $\sin ^{2} 2 \theta_{R} x$, with $x \sim 1$ if $\Delta m \gtrsim \Gamma$ and $x \ll 1$ if $\Delta m \ll \Gamma$. The decay $\mu \rightarrow e \gamma$ places an upper limit on $\sin 2 \theta_{R} \Delta m / m$ (Eqn.(四) so that there is competition between the two probes of flavor violation. Thus, in order for the signal at the LHC to be significant in the region of the $\left(\sin 2 \theta_{R}, \Delta m / m\right)$ plane beyond the reach of the $\mu \rightarrow e \gamma$ limit, there should be a range of $\Delta m / m$ where $\Delta m \gtrsim \Gamma$ so that $x \sim 1$ and $\Delta m / m$ is small enough (for a given value of $\sin 2 \theta_{R}$ ) so that $\mu \rightarrow e \gamma$ is suppressed. It will be seen that for $\Delta m / m \sim \Gamma / m$ (so that $x \sim 1$ ), at this LHC Point, $\sin 2 \theta_{R}$ is unconstrained by the $\mu \rightarrow e \gamma$ limit, affording the LHC the opportunity to either detect a signal or extend the limit.

At this Point $\chi_{1}^{0} \approx \tilde{B}^{0}$. A computation of the one-loop $\tilde{B}^{0}$ contribution gives

$$
\sin 2 \theta_{R} \frac{\Delta m_{R}^{2}}{\tilde{m}_{R}^{2}}\left(\frac{100 \mathrm{GeV} \tilde{m}_{R}}{M_{\chi_{1}^{0}}^{2}}\right)^{2} 20 F\left(\alpha_{L}, \alpha_{R}, t\right)<0.013 \times \sqrt{\frac{B R(\mu \rightarrow e \gamma)}{4.9 \times 10^{-11}}} .
$$

Here $\alpha_{H}=\tilde{m}_{H}^{2} / M_{\chi_{1}^{0}}^{2}(H=L, R), t=(A+\mu \tan \beta) / \tilde{m}_{R}$,

$$
F\left(\alpha_{L}, \alpha_{R}, t\right)=H\left(\alpha_{R}\right)+t \alpha_{R}^{1 / 2} \frac{\partial K}{\partial \alpha_{R}}\left(\alpha_{L}, \alpha_{R}\right)
$$

with

$$
K(x, y)=\frac{g(x)-g(y)}{x-y}, g(x)=\frac{1+2 x \log x-x^{2}}{2(x-1)^{3}}
$$

and

$$
H(x)=\frac{-x^{3}+9 x^{2}+9 x-17-(6+18 x) \log x}{6(x-1)^{5}} .
$$

Two useful facts are $H(1)=\frac{\partial}{\partial y} K(1,1)=-1 / 20$; hence the factor of 20 on the left side of Eqn.(41). At this LHC Point, $m_{\tilde{l}_{R}} \sim 160 \mathrm{GeV}, m_{\chi_{1}^{0}} \sim 120 \mathrm{GeV}$, and $m_{\tilde{l}_{L}} \sim 240 \mathrm{GeV}$. Inputing these masses into the above formula simplifies it to:

$$
\frac{\sin 2 \theta_{R}}{0.39} \times \frac{\Delta m_{R}}{\tilde{m}_{R}} \times(1+0.48 t)<0.03 \times \sqrt{\frac{B R(\mu \rightarrow e \gamma)}{4.9 \times 10^{-11}}} .
$$

At this Point $t \approx 5-10$. However, a larger variation in $t$ is allowed without affecting the flavor violating signal, since both $A$ and $\operatorname{sgn}(\mu)$ do not qualitatively affect the 4-lepton event rate $\square$. In any case, the values $\sin 2 \theta \approx 0.39$ and $\Delta m_{R} \sim \Gamma$ (so that $x \sim 1$ ) with a typical value of $\Gamma \sim \alpha_{e m} m \sim 10^{-2} \times \tilde{m}_{R}$ are consistent with $\mu \rightarrow e \gamma-$ recall that $\sin 2 \theta \approx 0.39$ and

\footnotetext{
${ }^{21}$ It is important to maintain the relation $m_{\chi_{2}^{0}}>m_{\tilde{e}_{R}}$ though.
} 
$x \sim 1$ was assumed to obtain the estimate of $S_{F V}$ in Eqn.(30). In fact, at this LHC Point $\Gamma \sim 125 \mathrm{MeV}$ [9], so that $\Gamma / m \sim 8 \times 10^{-4}$ which is smaller than $\alpha_{e m}$. So for $\Delta m / m \gtrsim$ $2 \Gamma / m \approx 1.6 \times 10^{-3}$, it follows that $x \sim 1$. From Eqn.(45) and for maximal mixing $\left(\sin 2 \theta_{R}=1\right.$ ), $\Delta m / m<0.39 \times 0.03 /(1+0.48 t) \approx 4 \times 10^{-3}$ (for $t \approx 5$ ). Thus for $1.6 \times 10^{-3} \lesssim \Delta m / m \lesssim 4 \times 10^{-3}$ and $\sin 2 \theta_{R}=1, \mu \rightarrow e \gamma$ is satisfied and $x \sim 1$. So at this Point even for maximal mixing there is a large range of $\Delta m / m$ for which $x \sim 1$ and $\mu \rightarrow e \gamma$ is safe. Of course, smaller mixing could be probed by the LHC, in which case the upper bound on $\Delta m / m$ allowed by $\mu \rightarrow e \gamma$ is larger. In this case for a given $\sin \theta_{R}$ there is a larger range of $\Delta m / m$ for which $x \sim 1$ (so that there is no suppression of the LHC signal) and $\mu \rightarrow e \gamma$ is safe.

\section{Conclusions}

We believe that it is possible to detect SUSY lepton flavor violation at the LHC using events with 4 leptons from the cascade decays of squarks provided the following conditions are satisfied:

0 . Either $R$-parity is conserved or $\chi_{1}^{0}(L S P)$ decays outside the detector,

1. $\chi_{2}^{0}$ pair production in cascade decays of squarks is large and $\chi_{2}^{0}$ has a large decay branching fraction to $\tilde{l}^{*} l$ (to get enough statistics),

2. Hadronic decays of $\tau \mathrm{s}$ can be detected with a known efficiency so that the background from the $\chi_{2}^{0} \rightarrow \tau \tau$ decay can be estimated,

3. The $b$-jet detection efficiency is good so that the background from events with stop/sbottom can be rejected,

4. The stop/sbottom production rate, either direct or in gluino decays, is not too much larger than the production of first two generation squarks,

5. The first two generation squarks decay largely to $\chi_{2}^{0}, \chi_{1}^{0}$ and $\chi_{1}^{ \pm}$, so that the background to flavor violating 4 lepton events from decays of heavier neutralinos/chargino to $W \mathrm{~s}$, lighter chargino, heavier sleptons etc. is small. This condition can be realised in the so-called gauginolike region,

6. The mass splitting is $\Delta m \sim \Gamma$ or larger, so there is no suppression of the signal due to the quantum interference effect.

The arguments presented here are clearly semi-quantitative, and further study requiring a detailed simulation of these processes is required. This is beyond the scope of this work.

\section{Acknowledgements}

We thank Nima Arkani-Hamed and Mahiko Suzuki for discussions, and Ian Hinchliffe in particular for discussions and allowing us to use the simulations of Point 5 of the LHC study. This work was supported in part by the U.S. Department of Energy under Contract DE-AC03-76SF00098. 
KA thanks Frank Paige for discussions. MG thanks the Natural Sciences and Engineering Research Council of Canada for their support.

\section{References}

[1] N.V. Krasnikov, Phys.Lett. B388, 783 (1996).

[2] N. Arkani-Hamed et al., Phys. Rev. Lett. 77, 1937 (1996).

[3] N. Arkani-Hamed et al., Nucl. Phys. B505, 3 (1997).

[4] N.V. Krasnikov, JETP Lett. 65, 148 (1997).

[5] S. I. Bityukov and N.V. Krasnikov, hep-ph/9712358.

[6] See, for example, N. Arkani-Hamed, H.-C. Cheng and L.J. Hall, Nucl. Phys. B472, 95 (1996), and references therein.

[7] H. Baer et al., Phys. Rev. D49, 3283 (1994).

[8] A. Bartl et al., Proceedings of the 1996 DPF Summer Study (Snowmass, CO, 25 June - 12 July, 1996), LBNL-39413 (1996).

[9] I. Hinchliffe et al., Phys. Rev. D55, 5520 (1997) and private communication with I. Hinchliffe.

[10] F. Paige, communication at the conference.

[11] Y. Coadou et al., ATLAS Internal Note ATL-PHYS-98-126, LBNL-41757.

[12] See p.191-192 of chap.12 (Physics Performance) of the CMS Technical Proposal at http://cmsinfo.cern.ch/TP/TP.html.

[13] See Fig.3.42, p.98 of ATLAS Collaboration, Technical Proposal, CERN/LHCC/94-43, LHCC/P2 (1994). 